\title{
A TRIGONOMETRIC INEQUALITY AND ITS GEOMETRIC APPLICATIONS
}

\author{
FARUK F. ABI-KHUZAM
}

Abstract. A sharp inequality for a linear combination of four cosines is obtained. It is used to sharpen inequalities due to Florian, Lenhard, Ozeki all of which extend the classical ErdösMordell inequality. Reverse isoperimetric inequalities for cyclic quadrilaterals are also obtained.

Mathematics subject classification (1991): 51M16.

Key words and phrases: Erdös-Mordell inequality.

\section{REFERENCES}

[1] Bottema O., Djordjevic R. Z., Janic R. R., Mitrinovic D. S., Vasic P. M., Geometric Inequalities, Wolters-Noordhoff publishing Groningen, 1969, The Netherlands.

[2] CoXeter H. S. M., GreitZer S. L., Geometry Revisited, The Mathematical Association Of America, 1967.

[3] Mitrinovic D. S., PeCaric J. E., Volenec V., Recent Advances In Geometric Inequalities, Kluwer Academic publishers, 1989.

[4] Florian A., Zu einem Satz von P. Erdos, Elem. Math. 13 (1958), 55-58.

[5] LENHARD H-C., Verallgemeinerung und Verscharfung der Erdos-Mordellschen Ungleichung fur Polygone, Arch. Math. Vol. XII, 1961, 311-314. 